Article

\title{
Fractal Dimension Based Carotid Plaque Characterization from Three-Dimensional Ultrasound Images
}

\author{
Ran Zhou ${ }^{1,2}$, Mingyue Ding ${ }^{1,2, *}$, Yongkang Luo ${ }^{1,2}$ and Aaron Fenster ${ }^{3}$ \\ 1 Medical Ultrasound Laboratory, College of Life Science and Technology, Huazhong University of Science \\ and Technology, Wuhan 430074, China; RanZhou927@163.com (R.Z.); myding@mail.hust.edu.cn (Y.L.) \\ 2 Key Laboratory of Molecular Biophysics of Education Ministry of China, College of Life Science and \\ Technology, Huazhong University of Science and Technology, Wuhan 430074, China \\ 3 Imaging Research Laboratories, Robarts Research Institute, Western University, \\ London, ON N6A 3K7, Canada; afenster@imaging.robarts.ca \\ * Correspondence: myding@mail.hust.edu.cn; Tel.: +86-27-8779-2366
}

\begin{abstract}
Carotid atherosclerotic lesions are a major cause of cerebrovascular disease (CVD). Identification and quantification of carotid plaques are important for categorizing the vulnerability of plaques for rupture and assessing the impact of treatments. The irregularity of plaque surface is associated with previous plaque rupture and plays an important role in the risk of stroke. Thus, the aim of this study is to develop and validate novel vulnerability biomarkers from three-dimensional ultrasound (3DUS) images by analyzing the surface morphological characterization of carotid plaque using fractal geometry features. 3D box-counting and 3D blanket are the two types of 3D fractal dimension that were employed to describe the smoothness of plaques. This fractal dimension analysis tool was used to evaluate the effect of atorvastatin using 3DUS carotid images, which were acquired from 6 patients treated with atorvastatin with $80 \mathrm{mg}$ daily and 5 patients with placebo. The Student's T Test results showed that those two fractal features were effective for detecting the statin-related changes in carotid atherosclerosis with $p<0.0068$ and $p<0.015$ respectively, suggesting that 3D fractal dimension measurements can be used effectively to analyze the surface characteristics of carotid plaques, especially for evaluating the impact of the treatment.
\end{abstract}

Keywords: fractal dimension; carotid plaque characterization; three dimensional ultrasound; an evaluation of the vulnerability of the plaques

\section{Introduction}

Carotid plaques are a major cause of cerebrovascular disease (CVD) and their rupture is widely viewed as the leading cause leading to stroke. The statistical reports from the World Health Organization (WHO) show that an estimated 17.5 million people died from CVDs in 2012, representing $31 \%$ of all global deaths. Of these deaths, an estimated 7.4 million were due to coronary heart disease and 6.7 million were due to stroke [1]. Thus, identification and quantification of vulnerable carotid plaques are extremely important for monitoring symptomatic patients at risk of stroke and assessing the impact of treatments [2]. Imaging-based assessments of atherosclerosis using as noninvasive measurement tools based on computed tomography (CT), magnetic resonance imaging (MRI) and ultrasound (US) have been widely accepted for diagnosis and monitoring of patients at risk of stroke. However, ultrasound is the most preferred modality for identifying vulnerable plaques due to its non-invasiveness, low cost and effectiveness [3].

Some early studies using B-mode ultrasound images of carotid arteries primarily focused on statistical measurements [4-6], such as the gray-scale medial (GSM), the mean, the median, the standard deviation etc. Recently, texture features were extensively used for the characterization of carotid plaques, which have been shown to have considerable success in identifying symptomatic and asymptomatic plaques. The most commonly used texture features were included: spatial gray 
level dependence matrices (SGLDM), gray level difference statistics (GLDS), neighborhood gray tone difference matrix (NGTDM), laws texture energy measures, local binary pattern (LBP), Fourier power spectrum and fractal-dimension texture analysis [7-9].

Three-dimensional (3DUS) carotid ultrasound imaging improves the visualization and quantification of carotid plaque as it provides a more accurate, repeatable and effective measurement tool to analyze plaque composition and morphology, as well as monitor treatment effects on atherosclerosis [10]. Previously, Landry and Fenster proposed to use the carotid plaque volume measurements (TPV) from 3DUS as a phenotype of carotid atherosclerosis [11-13]. More recently, Wannarong and Parraga reported the first result comparing progression of intima-media thickness (IMT), total plaque area (TPA), and TPV in clinical practice [14]. To overcome the limitations of TPV, such as image interpretation and measurement differences within and between observers, long training times for observers and long duration to perform the manual segmentation were required. Egger and Spence developed a more reproducible 3DUS measurement of carotid atherosclerosis vessel wall volume (VWV), which is a measurement of vessel wall thickness and plaque within the common carotid artery, the internal and external carotid branches [15]. Chiu and Egger used vesselwall-plus-plaque thickness (VWT) and VWT-Change values of the 3D carotid vessel wall to provide rich information on the spatial distribution of plaque burden, which is important in monitoring patients and in developing treatment strategies $[16,17]$. They also proposed a technique to flatten the $3 \mathrm{D}$ VWT and VWT-change maps in an area preserving manner, in order to facilitate the identification and quantification of a diseased region [18, 19].

Texture analysis of carotid artery atherosclerosis was also used to quantitatively evaluate local carotid arterial statin effects in 3DUS images, which was observed to be more sensitive in detecting statin-related changes in carotid atherosclerosis than VWV [20, 21]. Engelen and Wannarong utilized the 3D carotid ultrasound plaque texture to predict vascular events in patients at risk [22]. It was reported that irregularity of plaque significantly increased the risk of stroke [23]. Thus, Chiu and Beletsky identified the irregularity and ulcerations of carotid plaque surface ulcers from in vivo 3DUS images based on the mean and Gaussian curvatures [24]. Kuk and Wannarong quantified carotid total ulcer volume from 3DUS images to investigate the relationship of total ulcer volume to vascular events [25].

The purpose of our paper is to develop novel vulnerability biomarkers from 3DUS images by analyzing the surface morphological characterization of carotid plaques, since the irregularity of plaque surface is associated with previous plaque rupture and plays an important role in the risk of stroke $[26,27]$. Fractal geometry features can be used to identify the characterization of carotid plaques as a descriptor of volume-based texture and surface morphology of plaques. Asvestas and Golemati demonstrated that the fractal dimension could be used as a single determinant for the discrimination of symptomatic and asymptomatic subjects [28]. However, the fractal dimension feature was just used as a texture feature in [28], which demonstrated the roughness of its intensity surface from B-mode ultrasound images. In this paper, the fractal dimension applied to 3DUS images was used as not only a volume texture feature, but also a descriptor of the roughness of carotid plaque surfaces. Moreover, it is the first to estimate fractal dimension from 3DUS images of carotid plaque which could be an effective measure to describe the smoothness of carotid plaque surface and assess the impact of treatments.

\section{Materials and Methods}

\subsection{Subject and image acquisition}

\subsubsection{Study Subjects}

Thirteen 3DUS images of carotid plaque were acquired from eleven subjects with carotid stenosis over $60 \%$, among which 6 subjects with a mean age \pm SD of $68 \pm 8.6$ were treated with $80 \mathrm{mg}$ atorvastatin daily for three months and the remaining 5 patients with a mean age \pm SD of $70 \pm 9.4$ were supplied with the placebo. All subjects were recruited from The Premature Atherosclerosis 
Clinic and The Stroke Prevention Clinic at University Hospital (London Health Sciences Centre, London, Canada) and the Stroke Prevention and Atherosclerosis Research Centre in Western University (Robarts Research Institute, London, Canada).

\subsubsection{Image acquisition}

For all subjects, at baseline and 3 months later, both the left and right carotid arteries were scanned, using a Philips/ATL HDI 5000 US transducer with an L12-5 probe (8.5 MHz central frequency) attached to a motorized linear 3DUS acquisition system moving along the neck at a uniform speed of $3 \mathrm{~mm} / \mathrm{s}$.

The 3D images of carotid arteries were reconstructed from the set of 2D frames during the scan by 3D Quantify (a multi-planar visualization software) [29]. Figure 1 shows the 3D plaque manually segmented by an expert using this software.

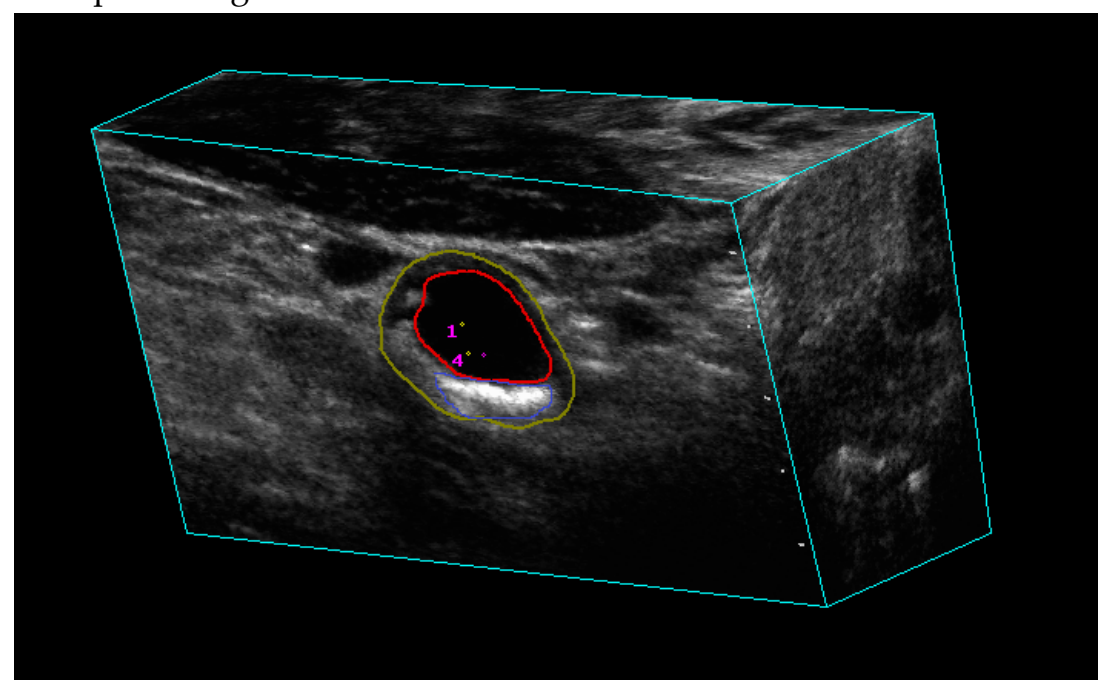

(a)

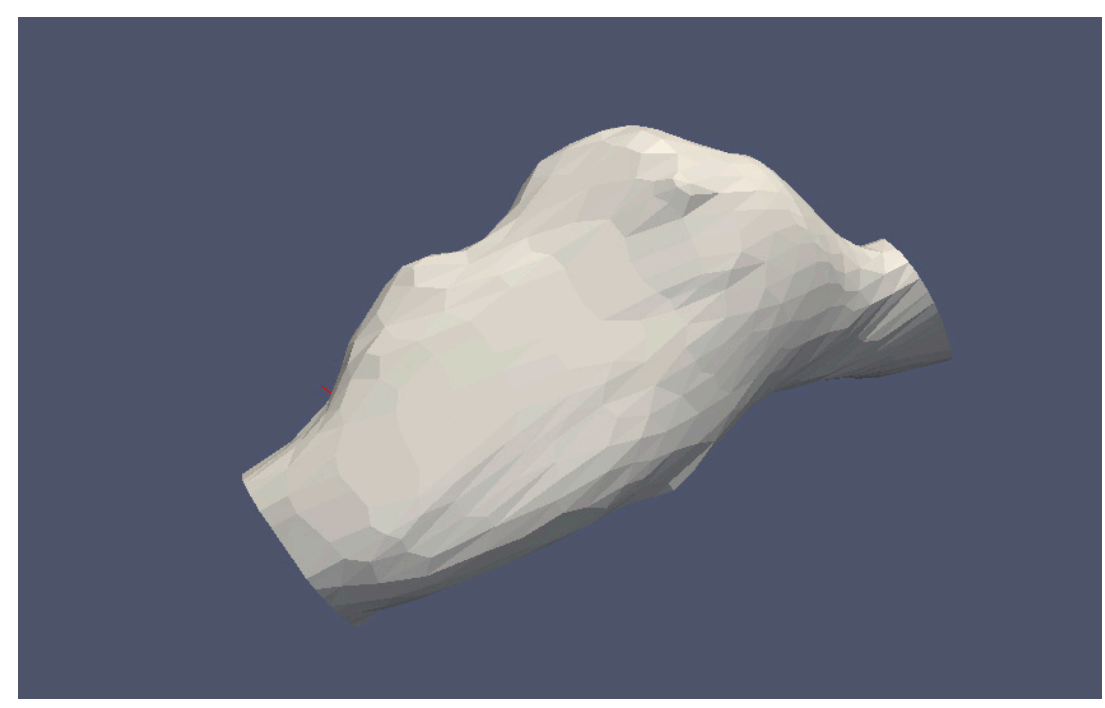

(b)

Figure 1. Result of segmenting plaque manually from 3D US image of carotid artery. (a) The plaque is manually segmented from 3DUS images. The red contour shows the lumen intima (the lumen boundary), the yellow contour shows the adventitia (the wall boundary) and the blue is a plaque. (b) A 3D plaque reconstructed from 3DUS image.

\section{2. $3 D$ fractal dimension measures}

Fractal geometry was first introduced and developed by Mandelbrot [30] as a useful tool to describe and quantify the inherent irregularity of objects. The fractal dimension is used to describe 
the self-similarity of fractals, which is a measure to quantify the roughness of surfaces. There are a variety of algorithms to estimate fractal dimensions, such as box-counting method, variance method, blanket method, fractional-Brownian motion method, etc. The most popular among them is boxcounting method, which is an upper limit of the Hausdorff-Besicovich dimension [31]. In this paper, box-counting and blanket methods were improved and applied to 3DUS images, which presents the roughness of plaque surface and 3D texture from voxel neighborhoods, respectively.

\subsubsection{D box-counting method}

The traditional box-counting method was proposed by Mandelbrot, which works by repeatedly covering the fractal image with different sized boxes, and then the fractal dimension (FD) is estimated as the slope of the regression line of box number and box size, which is defined as

$$
\mathrm{FD}=\lim _{r \rightarrow 0} \frac{\log N(r)}{\log (1 / r)}
$$

where $N(r)$ denotes the number of boxes with size $r$.

In this paper, we improve the traditional box-counting method and apply it to 3DUS images, to quantify the roughness and irregularity of plaque surfaces. The 3DUS image is divided into the voxelboxes with size $l$, which are considered as the cubes formed by $l+1$ consecutive pixels in the three directions of the 3D matrix. Then, the voxels completely contained in the tissue being studied are classified as BLACK. Voxels completely outside of the tissue being studied are classified as WHITE. While the voxels are partially contained in the tissue being studied are classified as GREY, i.e. the tissue surface cuts across the voxel.

The steps of 3D fractal dimension calculation is represented as follows:

1. Construct voxel sizes changing from 1 to maximum voxel size (the size of the cube enclosing entire 3D image)

2. The numbers of voxels classified as BLACK, WHITE and GRAY are counted respectively.

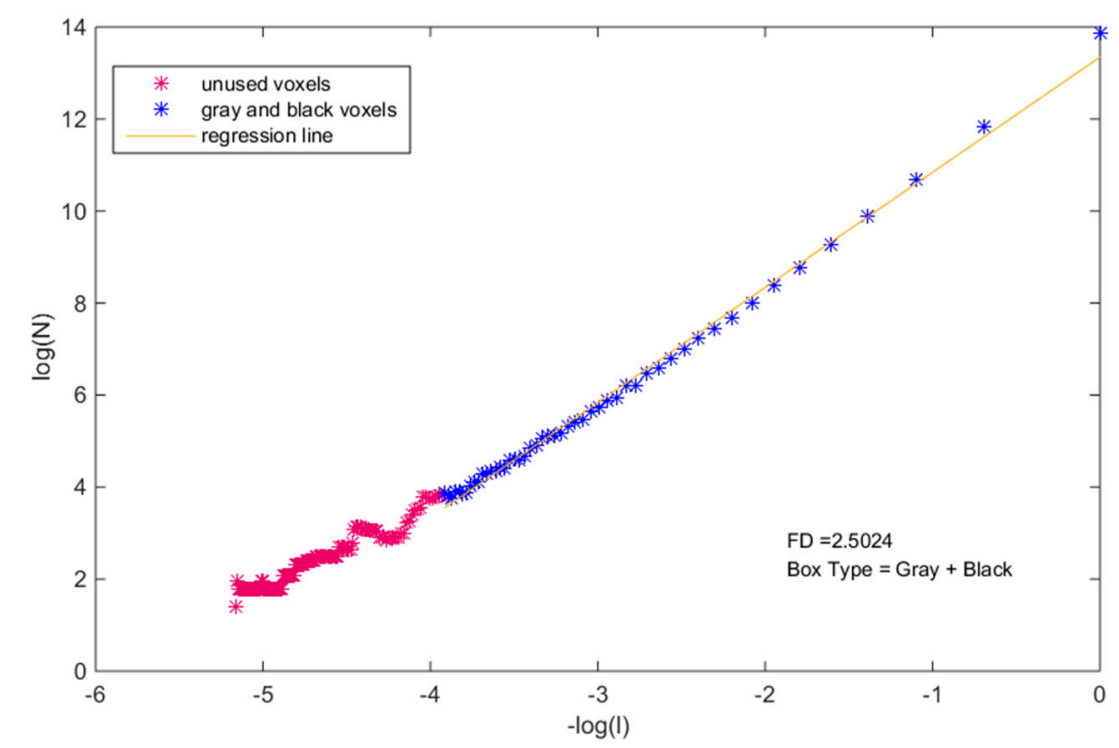

Figure 2. Linear regression results of 3D fractal dimension using box-counting method. Blue dots represent GRAY and BLACK voxels used in the linear regression and red dots are unused voxels. The slope of the red line shows the value of 3D fractal dimension of box-counting method.

3. The 3D FD is estimated from the least squares linear fit of $\log (\mathrm{N}(l))$ versus $\log (1 / l)$, where $\mathrm{N}(l)$ can be either the numbers of BLACK, GRAY, and WHITE voxel-boxes alone or combined. An example of this linear regression is shown in Figure 2.

4. The final value for $3 \mathrm{D}$ fractal dimension corresponds to the slope of linear regression. 


$$
\mathrm{FD}=-\lim _{l \rightarrow 0} \frac{\log (N(l))}{\log (l)}
$$

\subsubsection{D blanket method}

The blanket method was first introduced by Shmuel Peleg [32] for multiple resolution texture analysis and classification, which calculated texture features of intensity surface of 2D images.

To measure the length of a 2D curve, Mandelbrot [30] considered all points with distances to the coastline of no more than $\varepsilon$, which formed a strip of width $2 \varepsilon$, and the suggested length $L(\varepsilon)$ of the coast is the area of the strip divided by $2 \varepsilon$.

$$
L_{\varepsilon}=\frac{S}{2 \varepsilon}
$$

The Minkowski dimension is then equal to

$$
\mathrm{FD}=\lim _{\varepsilon \rightarrow 0}\left[D_{E}-\frac{\log \left(L_{\varepsilon}\right)}{\log (\varepsilon)}\right]
$$

where $D_{E}$ is the Euclidian dimension of the object, and $D_{E}$ is 1 for the curve.

Applied to the intensity surface of 2D images, the surface area is then the volume occupied by the blanket divided by $2 \varepsilon$.

$$
S_{\varepsilon}=\frac{V}{2 \varepsilon}
$$

The covering blanket is defined by its upper surface $u$, and its lower surface $b$. Initially, given the gray level function $g(i, j), u_{0}(i, j)=b_{0}(i, j)=g(i, j)$. For $\varepsilon=1,2,3, \ldots$, the blanket surfaces are defined as follows:

$$
\begin{gathered}
u_{\varepsilon}(i, j)=\max \left\{u_{\varepsilon-1}(i, j)+1, \max _{|(m, n)-(i, j) \leq 1|} u_{\varepsilon-1}(m, n)\right\} \\
b_{\varepsilon}(i, j)=\min \left\{b_{\varepsilon-1}(i, j)-1, \min _{|(m, n)-(i, j) \leq 1|} b_{\varepsilon-1}(m, n)\right\} \\
S_{\varepsilon}=\frac{\sum_{i, j}\left(u_{\varepsilon}(i, j)-b_{\varepsilon}(i, j)\right)}{2 \varepsilon}
\end{gathered}
$$

To extend blanket method to 3D voxel images, 3D fractal dimension presents texture feature of the intensity surfaces of the 3D voxel images. Let $g(x, y, z)$ be a 3D image and $u_{\varepsilon}, b_{\varepsilon}$ denote the top and bottom intensity surfaces respectively:

$$
\begin{gathered}
u_{\varepsilon}(i, j, k)=\max \left\{u_{\varepsilon-1}(i, j, k)+1, \max _{|(m, n, p)-(i, j, k) \leq 1|} u_{\varepsilon-1}(m, n, p)\right\} \\
b_{\varepsilon}(i, j, k)=\min \left\{b_{\varepsilon-1}(i, j, k)-1, \min _{|(m, n, p)-(i, j, k) \leq 1|} b_{\varepsilon-1}(m, n, p)\right\} \\
A_{\varepsilon}=\frac{\sum_{i, j, k}\left(u_{\varepsilon}(i, j, k)-b_{\varepsilon}(i, j, k)\right)}{2 \varepsilon}
\end{gathered}
$$

3D fractal dimension is given by the estimation of the slope $(p)$ of the linear fit of $\log \left(A_{\varepsilon}\right)$ against $\log (\varepsilon)$ and with the blanket's scale range from 1 to $\varepsilon$.

$$
\mathrm{FD}=\lim _{\varepsilon \rightarrow 0}\left[D_{E}-\frac{\log \left(A_{\varepsilon}\right)}{\log (\varepsilon)}\right]=D_{E}-\lim _{\varepsilon \rightarrow 0} \frac{\log \left(A_{\varepsilon}\right)}{\log (\varepsilon)}=3-p
$$




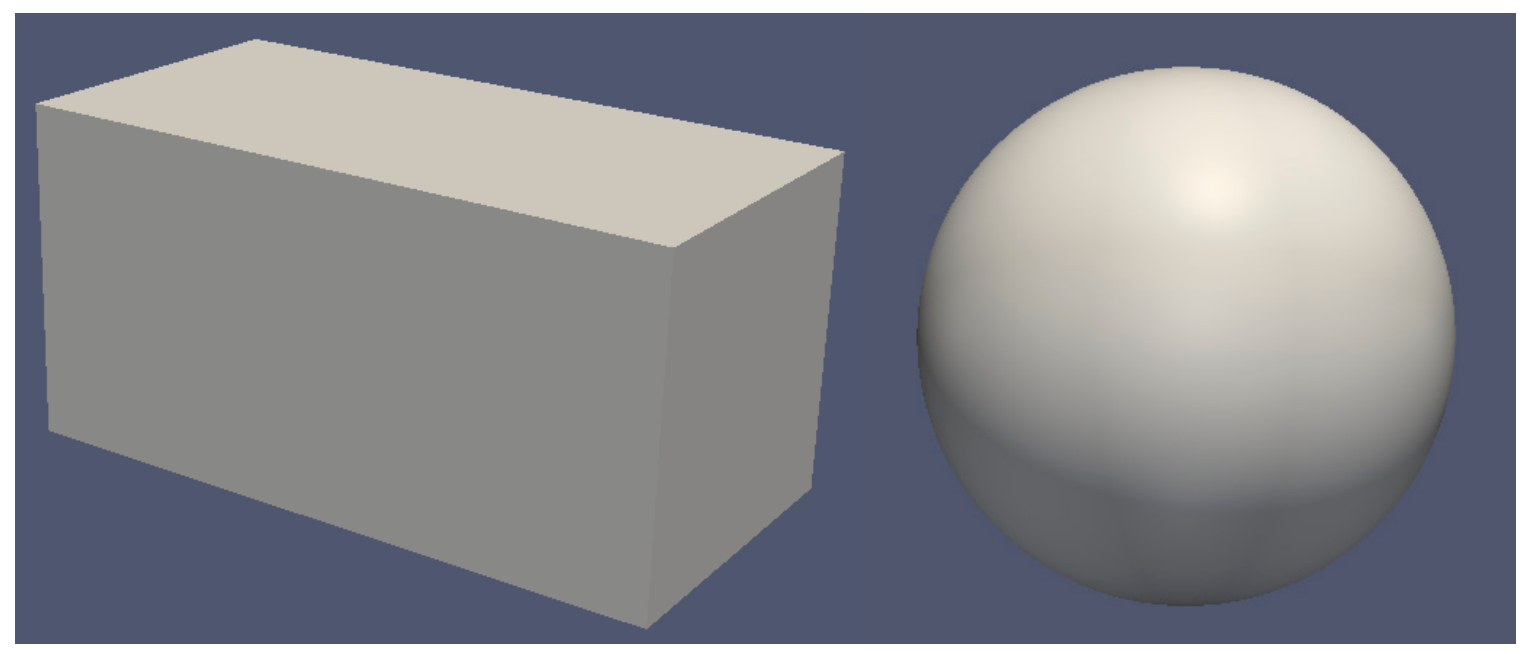

Figure 3. Synthetic cuboid and sphere for testing the validity and accuracy of the 3D fractal dimension calculations. The size of the cuboid is $160 \times 160 \times 320$, and the diameter of the sphere is 160 .

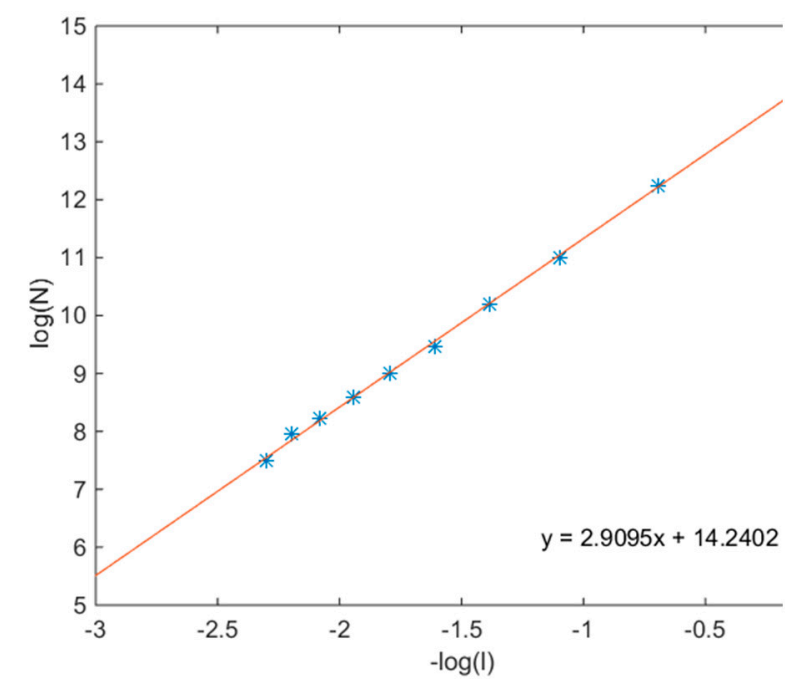

(a)

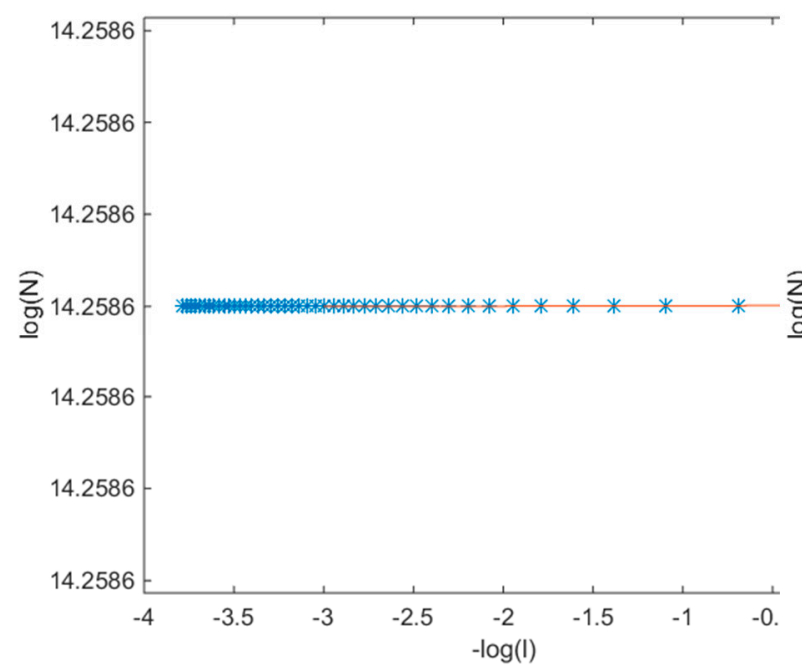

(c)

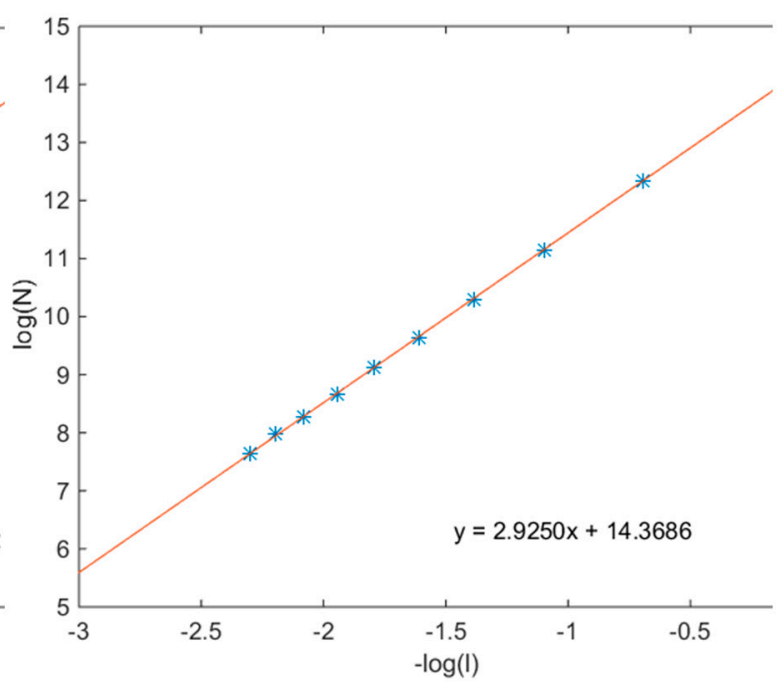

(b)

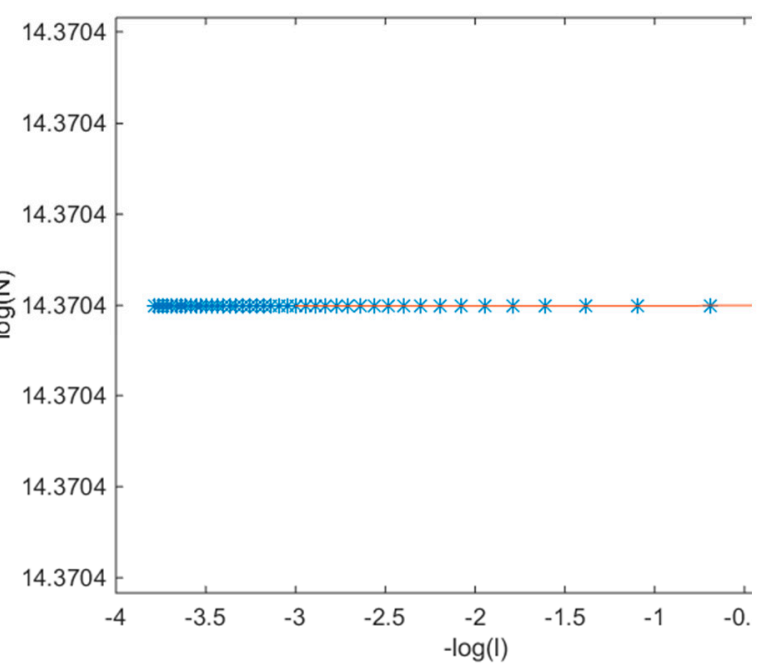

(d)

Figure 4. Linear regression results of calculating fractal dimensions using synthetic data. In (a) and (b), 3D box-counting method is applied, which shows the 3D fractal dimensions are 2.9095 and 2.9250 for cuboid and sphere data, respectively. In (c) and (d), 3D blanket method is used, and the 3D fractal dimensions are 3 for both cuboid and sphere data. 


\section{Results}

\subsection{Numerical accuracy of the $3 D$ fractal dimension calculations}

The 3D fractal dimension of 3D box-counting method and 3D blanket method were estimated using the synthetic cuboid and sphere data, as shown in Figure 3. In the experiments, the sum of BLACK and GRAY voxels has been used in all cases. Figure 4 shows the linear regression results of calculating fractal dimension. The 3D FDs are 2.9095 and 2.9250 for cuboid and sphere, respectively, using 3D box-counting method, and are 3.000016 and 3.000027 using 3D blanket method. These indicates that all fractal dimensions are very close to the theoretical values of 3 , which verifies the improved 3D fractal dimension computation methods is correct and valid.

\subsection{D fractal dimension of clinical data}

There were 11patients enrolled in the study and 14 plaques were manually segmented from the carotid artery 3DUS images by experts. All the 3D plaque images were interpolated by bicubic interpolation in order to make the number of axial slices matched the size of plaque in 2D US images. For instance, a plaque which contained 18 axial slices with a matrix of $99 * 67$ pixels was interpolated to $99^{*} 67^{*} 67$.

Both the 3D box-counting and 3D blanket methods were applied to all subjects, at baseline and 3 months later, to calculate the 3D fractal dimensions, and the Student's T Test was used to verify whether those two fractal features were effective for detecting the statin-related changes in carotid atherosclerosis.

\subsubsection{Results of 3D box-counting method}

Table 1 shows the 3D fractal dimension value using 3D box-counting method. For regular 3D objects with smooth surface, such as a sphere, the 3D fractal dimension is very close to the theoretical values of 3 . Thus, the fractal dimensions of 3D box-counting method are less than 3 for irregular 3D objects, and for greater the surface roughness, the 3D FD will be smaller than 3 . The results in Table 1 shows that the fractal dimension of plaque surfaces increases for subjects treated with atorvastatin during three months and the value decreases for subjects in control group, which demonstrates that the surface of plaques became more smooth after treatment with atorvastatin. Furthermore, the Student's T Test shows that there are statistical significant differences in the changes of 3D FDs between the two groups with $\mathrm{p}<0.0068$. This suggests that the smoothness of plaque surface, measured by fractal dimension of 3D box-counting method, can be used effectively to analyze carotid plaque characterics, especially for evaluating the impact of the treatment using atorvastatin.

Table 1. Fractal dimension values of 3D box-counting method.

\begin{tabular}{ccccccccc}
\hline \multicolumn{7}{c}{ Subjects treated with atorvastatin } \\
\hline Subjects & $\mathbf{1}$ & \multicolumn{2}{c}{$\mathbf{2}$} & $\mathbf{3}$ & $\mathbf{4}$ & $\mathbf{5}$ & $\mathbf{6}$ \\
\hline FDs (before) & 1.9060 & 2.1972 & 2.2948 & 2.1233 & 2.0036 & 2.0829 & 2.1750 & 2.2202 \\
\hline FDs (after) & 1.9296 & 2.3520 & 2.3372 & 2.2160 & 2.0391 & 2.1013 & 2.2188 & 2.1920 \\
\hline FDs change & -0.0236 & -0.1549 & -0.0425 & -0.0927 & -0.0354 & -0.0184 & -0.0438 & 0.0282 \\
\hline
\end{tabular}

FDs (before) are fractal dimension calculated before the subjects treated with placebo and atorvastatin. FDs (after) are calculated after the subjects treated with placebo and atorvastatin.

\begin{tabular}{ccccccc}
\hline \multicolumn{7}{c}{ Subjects treated with placebo } \\
\hline Subjects & $\mathbf{1}$ & $\mathbf{2}$ & $\mathbf{3}$ & $\mathbf{4}$ & \multicolumn{5}{c}{$\mathbf{5}$} \\
\hline FDs (before) & 2.2469 & 2.0815 & 2.2875 & 2.1129 & 2.2067 & 2.1060 \\
\hline FDs (after) & 2.2626 & 2.0391 & 2.1464 & 2.0279 & 2.1020 & 2.1242 \\
\hline FDs change & -0.0157 & 0.0423 & 0.1411 & 0.0850 & 0.1046 & -0.0182 \\
\hline
\end{tabular}




\subsubsection{Results of 3D blanket method}

The fractal dimension of 3D blanket method presents volume texture feature of 3D plaque images. Table 2 shows the results of 3D blanket fractal dimension of 3D plaque ultrasound images for the patients treated with atorvastatin and placebo respectively. The fractal dimension values of plaques increased for patients who were treated with atorvastatin during three months. On the contrary, the values of the control group decreased. Moreover, the results demonstrate that the volume texture of plaques changed significantly for the patients treated with atorvastatin. And in the Student's T Test, $\mathrm{p}$ is less than 0.015 for the volume texture changes of the plaque 3DUS images between the objects treated with atorvastatin and placebo. This result implies that fractal dimension measure of 3D blanket method is effective for detecting changes in carotid atherosclerosis after therapy.

Table 2. Fractal dimension values of 3D blanket method

\begin{tabular}{ccccccccc}
\hline \multicolumn{8}{c}{ Subjects treated with atorvastatin } \\
\hline Subjects & $\mathbf{1}$ & \multicolumn{2}{c}{$\mathbf{2}$} & $\mathbf{3}$ & $\mathbf{4}$ & $\mathbf{5}$ & $\mathbf{6}$ \\
\hline FDs (before) & 3.3412 & 3.3280 & 3.2560 & 3.2164 & 3.4663 & 3.3209 & 3.3143 & 3.3874 \\
\hline FDs (after) & 3.3557 & 3.3316 & 3.2975 & 3.2280 & 3.5235 & 3.3279 & 3.3274 & 3.4199 \\
\hline FDs change & -0.0145 & -0.0035 & -0.0415 & -0.0116 & -0.0572 & -0.0070 & -0.0131 & -0.0325 \\
\hline
\end{tabular}

\begin{tabular}{ccccccc}
\hline \multicolumn{7}{c}{ Subjects treated with placebo } \\
\hline Subjects & 1 & 2 & 3 & 4 & \multicolumn{2}{c}{5} \\
\hline FDs (before) & 3.2528 & 3.3589 & 3.2099 & 3.4219 & 3.2823 & 3.3200 \\
\hline FDs (after) & 3.2318 & 3.3390 & 3.2070 & 3.4042 & 3.3154 & 3.3008 \\
\hline FDs change & 0.0210 & 0.0198 & 0.0029 & 0.0178 & -0.0330 & 0.0192 \\
\hline
\end{tabular}

\section{Discussion}

In this study, a novel vulnerability biomarker, fractal dimension, was employed to analyze the morphological characterization of carotid plaque from 3D US images. Since irregularity of plaque surface plays an important role in the risk of stroke, the box-counting method was improved to describe the roughness of plaque surface. In addition, the 3D blanket method was used to describe the volume texture feature of the intensity surface of 3D US images.

Before performing the experiments using clinical data, both 3D box-counting and 3D blanket methods were applied to the synthetic cuboids and spheres data, and the 3D fractal dimensions of both method approached the value 3 , which verified that the numerical accuracy of fractal dimension computation is correct. Then, the algorithms were applied to 3D US images of carotid plaques obtained from two groups of the patients who were treated with atorvastatin and placebo respectively for 3 months. We compared the changes of 3D fractal dimension between the patients treated with atorvastatin and placebo for 3 months. For 3D box-counting method, the changes of 3D fractal dimensions increased significantly $(p<0.0068)$, which implied that the surface of carotid plaques became smooth after treatment with atorvastatin. On the contrary, the roughness of carotid plaque surfaces had no obvious change and some even became much rougher for the control group supplied with placebo. For the 3D blanket method, the changes of fractal dimensions between the patients at baseline and 3 months later indicated that volume texture feature changes significantly for treatment with atorvastatin $(\mathrm{p}<0.015)$. This result demonstrates that the $3 \mathrm{D}$ fractal dimension measure, using box-counting method and blanket method, can describe the characteristics of carotid plaque from 3D US images, especially for assessing the impact of treatments with atorvastatin.

In this paper, we first applies the fractal dimension to 3D US images of carotid plaque and demonstrated that the 3D fractal dimension measure, using box-counting method and blanket method, could describe the roughness of plaque surfaces and the volume texture of plaques. Thus, these metrics may be used for monitoring of carotid atherosclerosis therapy using atorvastatin. 


\section{Acknowledgments:}

This work was financially supported by the National 973 project (Grant No. 2011CB933103) and partly supported by the Special Research Fund for the Doctoral Program of Higher Education (No. 20130142130006) and the Innovation Research Foundation of Huazhong University of Science and Technology (No. 2013ZZGH018).

Author Contributions: Ran Zhou and Mingyue Ding conceived and designed the experiments; Ran Zhou performed the experiments; Ran Zhou and Yongkang Luo analyzed the data; Aaron Fenster contributed analysis tools; Ran Zhou and Mingyue Ding wrote the paper.

Conflicts of Interest: The authors declare no conflict of interest.

\section{Abbreviations}

The following abbreviations are used in this manuscript:

3DUS: Three-dimensional ultrasound

CVDs: Cardiovascular disease

FD: Fractal dimension

\section{References}

1. World Health Organization. Available online: http://www.who.int/mediacentre/factsheets/fs317/en/ (accessed on 1, August, 2016).

2. Lechareas, S.; Yanni, A.E.; Golemati, S.; Chatziioannou, A.; Perrea, D. Ultrasound and Biochemical Diagnostic Tools for the Characterization of Vulnerable Carotid Atherosclerotic Plaque. Ultrasound in Med. E Biol. 2016, 42, 31-43.

3. U-King-Im, JM.; Young, V.; Gillard, J.H. Carotid-artery imaging in the diagnosis and management of patients at risk of stroke. Lancet Neurol 2009, 8, 569-80.

4. El-Barghouty, N.; eroulakos, G.; Nicolaides, A.; Androulakis, A.; Bahal V. Computer assisted carotid plaque characterization. Eur. J. Vasc. Endovasc. Surg. 1995, 9, 548-557.

5. Elatrozy, T. S.; Nicolaides, A. N.; Tegos, T. J.; Griffin, M. The objective characterisation of ultrasonic carotid plaque features. Eur. J. Vasc. Endovasc. Surg. 1998, 16, 223-230.

6. Picano, E.; Paterni, M. Ultrasound Tissue Characterization of Vulnerable Atherosclerotic Plaque. International Journal of Molecular Sciences 2015, 16, 10121-10133.

7. Niu, L.; Qian, M.; Yang, W.; Meng, L.; Xiao, Y.; Wong, Kelvin K. L.; Abbott D.; Liu, X.; Zheng, H. R. Surface Roughness Detection of Arteries via Texture Analysis of Ultrasound Images for Early Diagnosis of Atherosclerosis. Plos One 2013, 8, e76880.

8. Acharya, U.R.; Vinitha, S.S.; Muthu, R.K.M.; Molinari, F.; Saba, L.; Ho, S.Y.; Ahuja, A.T.; Ho, S.C.; Nicolaides, A.; Suri, J.S. Atherosclerotic risk stratification strategy for carotid arteries using texturebased features. Ultrasound Med Biol. 2012, 38(6), 899-915.

9. Tsiaparas, N.N.; Golemati, S.; Andreadis, I.; Stoitsis, J.S.; Valavanis, I.; Nikita, K.S. Comparison of multiresolution features for texture classification of carotid atherosclerosis from B-mode ultrasound. IEEE Trans. Inf. Technol. Biomed. 2011, 15(1), 130-7.

10. Tasneem, Z.; Naqvi, M.D. Quantifying Atherosclerosis by “3D” Ultrasound Works! : But There Is Work to Be Done. Journal of the American College of Cardiology 2015, 65, 1075-1077.

11. Landry, A.; Fenster, A. Theoretical and experimental quantification of carotid plaque volume measurements made by three-dimensional ultrasound using test phantoms. Med. Phys. 2002, 29, 2319 $-2327$.

12. Landry, A.; Spence, J.D.; Fenster, A. Measurement of carotid plaque volume by 3-dimensional ultrasound. Stroke 2004, 35:864-869.

13. Landry, A.; Spence, J.D.; Fenster, A. Quantification of carotid plaque volume measurements using 3D ultrasound imaging. Ultrasound Med. Biol. 2005, 31, 751-762.

14. Thapat Wannarong; Parraga, G.; Buchanan, D.; Fenster, A.; Andrew, A.; House; Daniel, G.; Hackam;Spence, J. D. Progression of Carotid Plaque Volume Predicts Cardiovascular Events. Stroke 2013, 44, 1859-1865 
15. Egger, M.; Spence, J.D.; Fenster, A.; Parraga, G. Validation of 3D ultrasound vessel wall volume: an imaging phenotype of carotid atherosclerosis. Ultrasound Med Biol. 2007, 33(6), 905-14. Epub 2007

16. Chiu, B.; Egger, M.; Spence, J.D.; Parraga, G.; Fenster, A. Quantification of carotid vessel atherosclerosis. Proceeding of the International Society for Optical Engineering (SPIE) 6143, 13 March 2006.

17. Chiu, B.; Egger, M.; Spence, J.D.; Parraga, G.; Fenster, A. Quantification of progression and regression of carotid vessel atherosclerosis using 3D ultrasound images. Proceedings of the 28th International Conference on IEEE/EMBS, New York City, USA, 2006.

18. Chiu, B.; Egger, M.; Spence J.D.; Parraga, G.; Fenster, A. Quantification of carotid vessel wall and plaque thickness change using 3D ultrasound images, Med. Phys. 2008, 35, 3691.

19. Chiu, B.; Egger, M.; Spence J.D.; Parraga, G.; Fenster, A. Area-preserving flattening maps of 3D ultrasound carotid arteries images. Medical Image Analysis 2008, 12, 676-688.

20. Awada, J.; Krasinsk, A.; Parraga, G.; Fenster, A. Texture analysis of carotid artery atherosclerosis from three-dimensional ultrasound images. Med. Phys. 2010, 37, 382-91.

21. Acharya, U.R.; Sree, S.V.; Krishnan, M.M.; Molinari, F.; Saba, L.; Ho, S.Y.; Ahuja, A.T.; Ho, S.C.; Nicolaides, A.; Suri, J.S. Atherosclerotic risk stratification strategy for carotid arteries using texturebased features. Ultrasound in Med. E Biol. 2012, 38, 899-915.

22. Engelen, A.; Wannarong, T.; Parraga, G.; Niessen, W. J.; Fenster, A.; Spence, J. D.; Bruijne, M. ThreeDimensional Carotid Ultrasound Plaque Texture Predicts Vascular Events. Stroke. 2014, 45, 2695-2701.

23. Rothwell, P.M.; Gibson, R.; Warlow, C.P. Interrelation between plaque surface morphology and degree of stenosis on carotid angiograms and the risk of ischemic stroke in patients with symptomatic carotid stenosis. On behalf of the European Carotid Surgery Trialists' Collaborative Group. Stroke 2000, 31, 615-621.

24. Chiu, B.; Beletsky, V.; Spence, J. D.; Parraga, G.; Fenster, A. Analysis of carotid lumen surface morphology using three-dimensional ultrasound imaging. Phys. Med. Biol. 2009, 54, 1149-1167.

25. Mariya Kuk; Wannarong T.; Beletsky, V.; Parraga, G.; Fenster, A.; Spence, J. D. Volume of Carotid Artery Ulceration as a Predictor of Cardiovascular Events. Stroke 2014, 45, 1437-1441.

26. Homburg, P. J.; Rozie, S.; van Gils, M.J.; van den Bouwhuijsen, Q. J.; Niessen, W. J.; Dippel, D. W.; et al. Association between carotid artery plaque ulceration and plaque composition evaluated with multidetector CT angiography. Stroke 2011, 42, 367-372.

27. Madani, A.; Beletsky, V.; Tamayo, A.; Munoz, C.; Spence, J. D. High-risk asymptomatic carotid stenosis: ulceration on 3D ultrasound vs TCD microemboli. Neurology 2011, 77, 744-750.

28. Asvestas, P.; Golemati, S.; Matsopoulos, G. K.; Nikita, K. S.; Nicolaides, A.N. Fractal dimension estimation of carotid atherosclerotic plaques from B-mode ultrasound: a pilot study. Ultrasound Med Biol. 2002, 28(9), 1129-36.

29. Fenster, A.; Blake, C.; Gyacskov Igor; Landry, A.; Spence, J. D. 3D ultrasound analysis of carotid plaque volume and surface morphology. Ultrasonics 2006, 44, e153-7.

30. Mandelbrot, B. B. The Fractal Geometry of Nature. Freeman, San Francisco, CA, 1982.

31. Gangepain, J.; Roques-Carmes, C. Fractal approach to two dimensional and three dimensional surface roughness. Wear 1986, 109, 119-126.

32. Peleg, S.; Naor, J.; Hartley, R.; Avnir, D. Multiple resolution texture analysis and classification. IEEE Trans. Pattern Anal. Mach. Intell. 1984, 6(4), 518-23.

(C) 2016 by the authors; licensee Preprints, Basel, Switzerland. This article is an open access article distributed under the terms and conditions of the Creative Commons by Attribution (CC-BY) license (http://creativecommons.org/licenses/by/4.0/). 